\title{
Biorremediación de suelo contaminado con 55000 y 65000 de aceite residual automotriz y fitorre- mediación con Sorghum bicolor inoculado con Burkholderia cepacia y Penicillium chrysogenum
}

Bioremediation of soil contaminated by waste motor oil in 55000 and 65000 and phytoremediation by Sorghum bicolor inoculated with Burkholderia cepacia and Penicillium chrysogenum

Sánchez-Yáñez Juan Manuel ${ }^{1}$ Alonso-Bravo Jennyfer Nayeli ${ }^{1}$, Dasgupta-Schuber Nabanita ${ }^{1}$ Márquez-Benavides Liliana ${ }^{2 *}$

\begin{tabular}{l}
\hline Datos del Artículo \\
\hline 1 Microbiología Ambiental, Instituto de \\
Investigaciones Químico Biológicas Ed.B- \\
1 Ciudad Universitaria, Col F.J. Mujica \\
S/N, Felicitas del Rio, 58000. Morelia, \\
Mich., México. \\
2 Manejo de Residuos Sólidos y Medio \\
Ambiente. Instituto de Investigaciones \\
Agrícolas, Pecuarias y Forestales, \\
Universidad Michoacana de San Nicolás \\
Hidalgo, Morelia, Michoacán México. \\
*Dirección de contacto: \\
Liliana Márquez-Benavides. \\
Manejo de Residuos Sólidos y Medio \\
Ambiente. Instituto de Investigaciones \\
Agrícolas, Pecuarias y Forestales, \\
Universidad Michoacana de San Nicolás \\
Hidalgo, Morelia, Michoacán México. \\
E-mail: lili.marquez@gmail.com \\
\\
\hline
\end{tabular}

\section{Palabras clave:}

Cometabolismo, rizomicroorganismos, fisiología vegetal, fitodegradación.

J Selva Andina Biosph. 2015; 3(2):86-94.

\section{Historial del artículo}

Recibido abril, 2015

Devuelto noviembre, 2015

Aceptado noviembre, 2015.

Disponible en línea, noviembre 2015.

\begin{tabular}{l}
\multicolumn{1}{c}{ Editado por: } \\
Selva Andina \\
Research Society \\
\hline Key words: \\
\hline Cometabolism, \\
rhizomicroorganisms, \\
plant physiology, \\
phytodegradation.
\end{tabular}

\section{Resumen}

En suelo una elevada concentración de aceite residual automotriz (ARA) una mezcla de hidrocarburos alifáticos y aromáticos, provoca pérdida de su fertilidad. Una solución es aplicar la remediación química que es costosa y causa contaminación colateral. En contraste una alternativa ecológica es la biorremediación (BR) por bioestimulación (BS), seguida de fitorremediación (FR) con Sorghum bicolor y microorganismos promotores del crecimiento vegetal (MPCV) para reducir el ARA a un valor inferior al máximo permisible por la NOM-138 SEMARNAT/SS-2003 de 4400 ppm/Kg de suelo. Los objetivos de esta investigación fueron: i) BR de suelo contaminado con 55000 y 65000 ppm de ARA por bioestimulación; y ii) FR con Sorghum bicolor inoculado con Burkholderia cepacia y Penicillium chrysogenum NOM138 SEMARNAT/SS-2003. Así en ese suelo la BS con una solución mineral (SM) y Vicia sativa como abono verde (AV) redujeron el ARA de 55000 a 33400 ppm y de 65000 a 24300 ppm. Posteriormente la FR con S. bicolor con B. cepacia y P. chrysogenum (MPCV), el ARA decreció de 33400 ppm a 210 ppm, y de 24300 a 360 ppm, ambos valores inferiores al máximo permisible por NOM-138 SEMARNAT/SS-2003. Comparado con el mismo suelo control negativo con ARA, sin BR y FR, ahí la atenuación natural no cambio la cantidad de ARA. Lo anterior apoya la integración de la BR/FR en suelo impactado con elevadas concentraciones de ARA, como una opción ecológica en sustitución de remediación química costosa y contaminante.

(C) 2015. Journal of the Selva Andina Biosphere. Bolivia. Todos los derechos reservados.

\section{Abstract}

In soil spill a high concentration of waste motor oil (WMO) it's causing lost soil fertility, which is solved by remediation, but is expensive and polluting, an ecological alternative is bioremediation (BR) by biostimulation follow by phytoremediation (PY) with Sorghum bicolor using Burkholderia cepacia and Penicillium chrysogenum, promoting growth plant microorganisms (PGPM) at concentration value below to the maximum according to NOM-138 SEMARNAT/SS2003 de $4400 \mathrm{ppm} / \mathrm{Kg}$ soil. The objectives of this research were a) bioremediation of soil contaminated by high WMO concentrations by biostimulation with mineral solution and Vicia sativa as green manure (GM), and subsequent b) phytoremediation by $S$. bicolor with $B$. cepacia and $P$. chrysogenum to reduce remaining WMO at concentration below to maximum according to NOM-138 SEMARNAT/SS-2003. The results showed that biostimulation with mineral solution and V. sativa reduced WMO from 55000 to 33400 ppm, and from 65000 to 24300 ppm. Follow by PY by $S$. bicolor with B. cepacia and P. chrysogenum decreased WMO from 33400 ppm to 210 ppm, and from 24300 ppm to 360 ppm, compared to soil as negative control in which WMO did not change by natural attenuation. This suggests that to integrate BR and PY is an ecological option instead to apply chemical technique expensive and causing environmental pollution. 


\section{Introducción}

En el mundo y México el suelo contaminado con hidrocarburos (HC) provenientes del petróleo, como el aceite residual automotriz (ARA) es considerado un problema ambiental grave. El ARA es una mezcla de moléculas insolubles de HC alifáticos, aromáticos, policíclicos, halogenados y metales pesados, generados durante el ciclo de lubricación de automotores proceso que evita el desgaste de la piezas del motor durante su funcionamiento (Dua et al. 2002). En suelo para minimizar una elevada concentración de ARA igual o mayor a 40000 ppm, se recomiendan primero métodos químicos, que tienen el inconveniente de su elevado costo y efectos secundarios ambientales negativos. Sin embargo una alternativa ecológica adecuada para solucionar este problema es la biorremediación (BR) mediante una bioestimulación (BS) doble y secuencial con compuestos inorgánicos y orgánicos de Nitrógeno (N), Fósforo $(\mathrm{P})$ y Potasio (K) (Smith et al. 1998, Graham et al. 1999) con la intención de reducir la concentración del ARA para permitir entonces la fitorremediación (FR) con una gramínea de tipo del Sorghum bicolor cuya capacidad de eliminar HC puede ser potenciada con géneros de microorganismos promotores de crecimiento vegetal (MPCV) como: Burkholderia cepacia y Penicillium chrysogenum ambos oxidantes de HC, similares a los componentes del ARA, además los MPCV contribuyen a un sano crecimiento vegetal. Para que en el suelo se disminuya el ARA, a un valor inferior al máximo permisible de $4400 \mathrm{ppm} / \mathrm{kg}$ de suelo, según la norma mexicana NOM-138 SEMARNAT/SS-2003 para considerarlo remediado e útil para su explotación agrícola.

Es posible por tanto evitar la remediación del suelo impactado con alta concentración de HC mediante 87 acciones integrales de BR y FR. Al respecto (Ferrera-Cerrato et al. 2007), reportaron la BR de un suelo contaminado con 50000 ppm de combustóleo por BS con SM y posteriormente la FR con Phaseolus coccineus. Los resultados mostraron que la BS del suelo y su FR minimizaron el combustóleo a 3500 ppm. Con la base a lo anterior, los objetivos de este trabajo fueron: a) la BR de un suelo contaminado con 55000 y 65000 ppm de ARA y su posterior b) FR con $S$. bicolor inoculado con B. cepacia y $P$. chrysogenum para disminuir el ARA restante a una concentración inferior al valor máximo permitido por la NOM-138 SEMARNAT/SS-2003.

\section{Materiales y métodos}

Esta investigación se realizó en el invernadero del laboratorio de Microbiología Ambiental del Instituto de Investigaciones Químico Biológicas de la UMSNH. En el invernadero las condiciones microclimáticas promedio fueron temperatura de $23.2{ }^{\circ} \mathrm{C}$, luminosidad de $450 \mathrm{~mol} \cdot \mathrm{m}^{-2} \cdot \mathrm{s}^{-1} \mathrm{y}$ humedad relativa de $67 \%$. El ensayo de biorremediación/fitorre mediación del suelo impactado con dos altas concentraciones de ARA se dividió en dos fases. En la primera se realizó la BS de la doble y secuencial BS para disminuir la concentración de ARA y permitir la segunda fase con la FR del suelo mediante la siembra de semilla de $S$. bicolor coinoculado con $B$. cepacia y $P$. chrysogenum para reducir el ARA remanente de la BS a un valor inferior al máximo permitido por la norma mexicana NOM-138 SEMARNAT/SS-2003.

El suelo utilizado para el ensayo se colecto del horizonte A, con una textura franco arenosa, pobre en 
materia orgánica de $1.5 \%$ y $\mathrm{N}$ orgánico de $39 \mathrm{Kg} / \mathrm{ha}$, y un pH 6.7 ligeramente ácido clasificado como de tipo laterítico, este suelo fue ubicado a los $19^{\circ} 39^{\prime}$ $27^{\prime}$ ' de latitud norte $100^{\circ} 19^{\prime}$ '59' de longitud oeste, con una altitud de 1820 msnm de un zona con clima templado en un terreno agrícola denominado "La cajita" de la Tenencia Zapata del municipio de Morelia, Mich., México sobre el km 5 de la carretera Morelia-Pátzcuaro, México. El suelo fue llevado al invernadero donde se solarizó a $70{ }^{\circ} \mathrm{C} / 48 \mathrm{~h}$ posteriormente se tamizó y contamino con 55000 y 65000 ppm de ARA. El ARA fue proveniente de un taller mecánico eliminado por haber cumplido su ciclo de un automotor, el ARA se disolvió en agua con detergente comercial al 1.0\% (p/v) (La Corona $\left.{ }^{\circledR}\right)$ para su emulsión y mezclado con el suelo (SánchezYáñez 2011). Después el suelo se colocó en el sistema hidropónico de jarras de Leonard (Figura 1). El ensayo se estableció bajo un diseño experimental completamente al azar con 6 tratamientos y 6 repeticiones (Sánchez-Yáñez 2007).

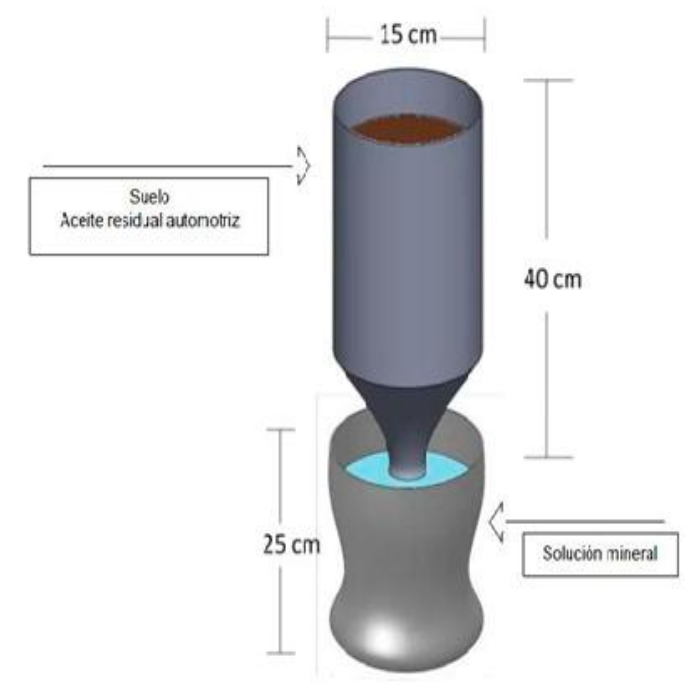

Figura 1 Diagrama de diseño de una jarra de Leonard

En la primera fase el suelo contaminado con 55000 y 65000 ppm de ARA y sin contaminar (control relativo), la primera bioestimulación se inició con la aplicación de la SM (en el reservorio de cada jarra de Leonard se agregaron $500 \mathrm{~mL} / 15$ días) con la siguiente composición química $(\mathrm{g} / \mathrm{L}) \mathrm{NH}_{4} \mathrm{NO}_{3}$ 10.0, $\mathrm{K}_{2} \mathrm{HPO}_{4}$ 2.5, $\mathrm{KH}_{2} \mathrm{PO}_{4}$ 2.0, $\mathrm{MgSO}_{4}$ 1.0, $\mathrm{NaCl} 0.1$, $\mathrm{CaCl}_{2}$ 0.1, $\mathrm{FeSO}_{4}$ (todos los reactivos empelados Simga-Aldrlch) trazas, ajustado a pH de 6.5 y 10.0 $\mathrm{mL}$ de solución de oligoelementos con la siguiente composición (g/L): $\mathrm{H}_{3} \mathrm{BO}_{3}$ 2.86, $\mathrm{ZnSO}_{4} 7 \mathrm{H}_{2} \mathrm{O}$ 0.22: $\mathrm{MnCl}_{2} 7 \mathrm{H} 2 \mathrm{O}$ 1.81; $\mathrm{K}_{2} \mathrm{MnO}_{4}$ 0.09; (todos los reactivos Sigma-Aldrich) ajustado a $\mathrm{pH}$ de 6.8. Mientras que en el suelo en las jarras de Leonard se mantuvo al $80 \%$ de capacidad de campo con agua potable por 14 semanas (Juárez-Cisneros \& Sánchez-Yáñez 2014. A continuación se inició la segunda bioestimulación en el mismo suelo contaminado con ARA y se fertilizo con la SM en el mismo suelo sin ARA o control relativo (CR), donde sembraron 10 semillas de $V$. sativa en macetas de $2 \mathrm{~kg}$, cuando la leguminosa alcanzó el nivel de plántula 20 días después de su siembra, cada planta se extrajo desde la raíz, se barbechó e incorporó en concentración del $5 \%(\mathrm{p} / \mathrm{p})$ en el suelo contaminado con ARA en las jarras de Leonard, luego se dejó mineralizar por 30 días y entonces se determinó la concentración de ARA por el método de Soxhlet (NMX-F-089-S1978) al igual que la concentración de Nitrógeno total (Sánchez-Yáñez 2007). Finalmente se inició la segunda fase, la FR de ese suelo para disminuir el ARA a un nivel no tóxico, para ello en el suelo de las jarras de Leonard se sembraron cuatro semillas de $S$. bicolor inoculadas con B. cepacia previamente cultivadas agar $P$. cepacia, ácido ázelaico triptamina o PCAT (g/L): $\mathrm{MgSO}_{4}$ 0.1, ácido ázaleico 0.20, triptamina $0.2 \mathrm{~g}$ (disuelto en $2.0 \mathrm{~mL}$ de alcohol absoluto): $\mathrm{K}_{2} \mathrm{HPO}_{4} 4.0, \mathrm{KH}_{2} \mathrm{PO}_{4}$ 4.0, extracto de leva- 
dura 0.02, (todos los reactivos Sigma-Aldrich) a un $\mathrm{pH}$ ajustado a 6.7, las cajas de PCAT se incubaron a 28-30 ${ }^{\circ} \mathrm{C}$ por $72 \mathrm{~h}$, en las cuales se agregaron 5.0 $\mathrm{mL}$ en solución salina estéril $(\mathrm{NaCl} 0.85 \%$ y detergente $0.01 \%$ ) para cosechar las células de B. cepacia. Entonces por cada 20 semillas de S. bicolor se empleó $1.0 \mathrm{~mL} \mathrm{B.} \mathrm{cepacia} \mathrm{con} \mathrm{aproximadamente}$ $600 \times 10^{6} \mathrm{UFC} / 1 \mathrm{~mL}$, posteriormente cuando $S$. bicolor alcanzó el nivel de plántula 22 días después de su siembra, se inocularon $3.0 \mathrm{~mL}$ a nivel del cuello de la planta con una suspensión de $P$. chrysogenum crecido en agar papa dextrosa por $72 \mathrm{~h} / 30^{\circ} \mathrm{C}$ cosechada con $5.0 \mathrm{~mL}$ de la solución salina estéril $(\mathrm{NaCl}$ $0.85 \%$ y detergente $0.01 \%$ ) equivalente a $1 \times 10^{6}$ propagulos/planta. El S. bicolor se alimentó con la SM descrita previamente por (Sánchez-Yáñez 2007) y cuando alcanzo el estado fisiológico floración bajo la premisa de que en esta etapa fisiológica la gramínea alcanza su máxima actividad metabólica para fitodegradar los HC del ARA, por ello en ese punto se tomó el suelo de rizósfera de S. bicolor cuantifico la concentración del ARA remanente por Soxhlet (Aurand et al. 1987). Todos los datos experimentales fueron analizados por Tukey al $0.05 \%$ (Hernández-Valencia \& Mager 2003, Ferrera-Cerrato et al. 2007)

\section{Resultados}

En la Tabla 1 se muestra la BR del suelo contaminado con 55000 y 65000 ppm de ARA por BS con $\mathrm{SM} / 14$ semanas e incorporación de $V$. sativa como AV/4 semanas, ahí se observa que el ARA se redujo desde 55000 a 33400 ppm, y de 65000 a 24300 ppm. En suelo las concentraciones de ARA fueron altas para lograr su reducción a un nivel inferior al máximo permisible por la NOM-138 SEMARNAT/SS2003 se continuo con la FR con S. bicolor inoculado con B. cepacia y P. chrysogenum (Juárez-Cisneros \& Sánchez-Yáñez 2014.

Tabla 1 Biorremediación de suelo con aceite residual automotriz por bioestimulación doble y secuencial con solución mineral y Vicia sativa como abono verde, sobre su concentración

\begin{tabular}{|c|c|c|c|c|}
\hline Fase 1 Suelo & $\begin{array}{c}\text { Concentración } \\
\text { inicial de ARA } \\
(\text { ppm) }\end{array}$ & $\begin{array}{c}\text { Final bi- } \\
\text { oestimulación } \\
(\mathbf{p p m})\end{array}$ & $\begin{array}{c}\text { Porcentaje (\%) de } \\
\text { Biorremediación }\end{array}$ & $\begin{array}{l}\text { Concentración de } \\
\text { nitrógeno total }\end{array}$ \\
\hline Control absoluto & 0.0 & $0.0^{\mathrm{C} *}$ & 0.0 & $0.63^{\mathrm{c}}$ \\
\hline $\begin{array}{l}\text { Aceite residual automotriz + } \\
\text { solución mineral + Vicia sativa }\end{array}$ & 55000 & $33400^{\mathrm{a}}$ & 39.28 & $0.69^{\mathrm{b}}$ \\
\hline $\begin{array}{l}\text { Control negativo aceite resi- } \\
\text { dual automotriz }\end{array}$ & 55000 & $55000^{\mathrm{b}}$ & 0.0 & - \\
\hline $\begin{array}{c}\text { Aceite residual automotriz + } \\
\text { solución mineral + Vicia sativa }\end{array}$ & 65000 & $24300^{a}$ & 62.61 & $0.56^{\mathrm{d}}$ \\
\hline $\begin{array}{c}\text { Control negativo aceite resi- } \\
\text { dual automotriz }\end{array}$ & 65000 & $65000^{\mathrm{b}}$ & 0.0 & - \\
\hline
\end{tabular}

*Tukey $(0.05)=$ Letras iguales $=$ sin diferencia estadística. 
Tabla 2 Fenología y biomasa de Sorghum bicolor inoculado con Burkholderia cepacia y Penicillium chrysogenum a floración usado para fitorremediar el suelo con aceite residual automotriz remanente de la bioestimulación

\begin{tabular}{|c|c|c|c|c|c|c|}
\hline \multirow[t]{2}{*}{ Sorghum bicolor ** } & \multirow{2}{*}{$\begin{array}{l}\text { Altura de } \\
\text { planta }(\mathrm{cm})\end{array}$} & \multirow{2}{*}{$\begin{array}{l}\text { Longitud } \\
\text { radical }(\mathrm{cm})\end{array}$} & \multicolumn{2}{|c|}{ Peso fresco (g) } & \multicolumn{2}{|c|}{ Peso seco (g) } \\
\hline & & & Aéreo & Radical & Aéreo & Radical \\
\hline Control absoluto (agua) & $43.30 \pm 2.95^{\mathrm{c} *}$ & $21.69 \pm 2.62^{\mathrm{cd}}$ & $7.41 \pm 1.95^{\mathrm{bc}}$ & $1.70 \pm 0.58^{\mathrm{c}}$ & $2.65 \pm 1.10^{\mathrm{c}}$ & $0.53 \pm 0.20^{\mathrm{c}}$ \\
\hline Control relativo & $54.17 \pm 9.70^{\mathrm{a}}$ & $21.18 \pm 2.62^{d}$ & $9.02 \pm 1.14^{\mathrm{ab}}$ & $1.64 \pm 0.5^{\mathrm{c}}$ & $3.91 \pm 0.64^{\mathrm{ab}}$ & $0.51 \pm 0.25^{\mathrm{c}}$ \\
\hline ARA 33400 ppm & $56.00 \pm 3.65^{a}$ & $25.84 \pm 2.30^{a b}$ & $9.70 \pm 1.19^{a}$ & $2.32 \pm 0.69^{b}$ & $4.91 \pm 1.29^{\mathrm{a}}$ & $1.11 \pm 0.31^{b}$ \\
\hline ARA 24300 ppm & $46.10 \pm 3.08^{\mathrm{b}}$ & $24.49 \pm 2.95^{b c}$ & $7.40 \pm 1.51^{\mathrm{bc}}$ & $1.74 \pm 0.64^{\mathrm{c}}$ & $2.55 \pm 1.04^{\mathrm{c}}$ & $0.59 \pm 0.26^{\mathrm{C}}$ \\
\hline
\end{tabular}

$*$ Tukey $(0.05)=$ Letras iguales $=$ sin diferencia estadística. ${ }^{* *}$ duración del ensayo 8 meses.

La fenología del S. bicolor inoculado con B. cepacia y $P$. chrysogenum para la FR del suelo con el ARA remanente y posterior la BS se muestra en la Tabla 2 , ahí el valor de la altura de planta de $S$. bicolor a floración fue de $56.0 \mathrm{~cm}$ en el suelo con 33400 ppm de ARA; mientras que $S$. bicolor en el suelo con 24300 ppm de ARA fue de $46.10 \mathrm{~cm}$; ambos valores sin diferencia estadística comparado con el valor de la altura de $S$. bicolor usado como control relativo (CR) de $54.17 \mathrm{~cm}$ y superior al de la altura de planta de $S$. bicolor empleado como control absoluto (CA) con $43.30 \mathrm{~cm}$; lo que sugiere un efecto fitotóxico del ARA remanente en la altura de $S$. bicolor cuando la concentración de ARA fue de 24300 (Smith et al. 1998).

Respecto a la longitud radical la Tabla 2 muestra que $S$. bicolor registró una de $28.7 \mathrm{~cm}$ en el suelo con 33400 ppm de ARA. Mientras que S. bicolor alcanzo una longitud radical de $25.84 \mathrm{~cm}$, en el suelo con 24300 ppm de ARA, estos valores fueron estadísticamente diferentes y superiores a la longitud radical de $S$. bicolor en el suelo sin ARA irrigado solo con agua CA con una longitud radical de 21.69 $\mathrm{cm}$ y de $S$. bicolor crecido en el suelo sin ARA ali- mentado con SM no inoculado con los MPCV (CR), ahí la longitud radical fue de $21.18 \mathrm{~cm}$.

En lo referente a la biomasa de S. bicolor usado en la FR del suelo para reducir el ARA remanente posterior, a la bioestimulación mostrada en la Tabla 2, en la cual se observó que el peso fresco aéreo de $S$. bicolor inoculado con B. cepacia y P. chrysogenumen en el mismo s suelo con 33400 ppm de ARA; ahí fue de $9.70 \mathrm{~g}$. Entre tanto en ese suelo pero 24300 ppm de ARA de peso fresco aéreo fue de $7.40 \mathrm{~g}$ el primer valor no registró diferencia estadística comparado con el peso fresco aéreo de $9.02 \mathrm{~g}$ del S. bicolor utilizado como CR sin inocular con los MPCV y alimentado con una SM, este valor tuvo diferencia estadística respecto al peso fresco aéreo de $7.41 \mathrm{~g}$ de $S$. bicolor empleado como CA irrigado solo con agua sin inocular en suelo no contaminada con ARA.

Respecto al peso fresco radical, S. bicolor con $B$. cepacia y $P$. chrysogenum en el suelo con 33400 ppm de $2.32 \mathrm{~g}$; mientras que en el suelo con 24300 ppm de ARA el peso fresco radical fue de $1.74 \mathrm{~g}$, ambos valores estadísticamente diferentes a los del S. bicolor usado como CA con $1.70 \mathrm{~g}$, así como al 
registrado en $S$. bicolor empleado como CR con $1.64 \mathrm{~g}$.

El peso seco aéreo de $S$. bicolor doble inoculado con B. cepacia y P. crysogenum fue de $4.91 \mathrm{~g}$ en el suelo con 33400 ppm de ARA, valor sin diferencia estadística comparado con el mismo valor en $S$. bicolor en el suelo CR sin ARA alimentado con SM con un peso seco aéreo de $3.91 \mathrm{~g}$.

La Tabla 3 se muestra la sumatoria de la biorremediación del suelo contaminado con las dos concentraciones de ARA: primero bioestimulado con la $\mathrm{SM}$, y en secuencia con la incorporación de V. sati- va como $\mathrm{AV}$, $\mathrm{y}$ finalmente su posterior fitorremediación para minimizar el ARA restante a un valor inferior al máximo aceptado por la NOM-138 SEMARNAT/SS-2003. Con la siembra de semilla de $S$. bicolor inoculada con B. cepacia y P. chrysogenum a floración. En el suelo con 33400 ppm la disminución del ARA fue de 210 ppm; en el mismo suelo con 24300 ppm de ARA se disminuyó hasta 360 ppm, en comparación con la concentración de ARA correspondiente de cada suelo empleado como control negativo; en los que la atenuación natural no cambió la cantidad ARA en los 8 meses del ensayo.

Tabla 3 Fitorremediación de suelo con Sorghum bicolor inoculado con Burkholderia cepacia y Penicillium chrysogenum para minimizar el aceite residual automotriz a un nivel inferior al máximo establecido por la norma mexicana NOM-138 SEMARNAT/SS-2003.

\begin{tabular}{|c|c|c|c|}
\hline Fase 2 Suelo & $\begin{array}{c}\text { Final bioestimulación } \\
\text { (ppm) }\end{array}$ & $\begin{array}{l}\text { Final Fitorremediación } \\
\quad \text { (floración) (ppm) }\end{array}$ & $\begin{array}{l}\text { Porcentaje de biorremediación } \\
\qquad(\%)\end{array}$ \\
\hline Control absolute & $0.0^{c *}$ & $0.0^{\mathrm{c}}$ & 0.0 \\
\hline $\begin{array}{l}\text { ARA + solución mineral }+V \text {. sativa } \\
\text { bicolor } \mathrm{L} \text {. inoculado con } \\
\text { B. cepacia y } P \text {. chrysogenum }\end{array}$ & $33400^{\mathrm{a}}$ & $210^{\mathrm{a}}$ & 99.61 \\
\hline Control negative & $55000^{\mathrm{b}}$ & $55000^{\mathrm{b}}$ & 0.0 \\
\hline $\begin{array}{l}\text { ARA + solución mineral }+V \text {. sativa } \\
\text { bicolor } \mathrm{L} \text {. inoculado con } \\
\text { B. cepacia y } P \text {. chrysogenum }\end{array}$ & $24300^{\mathrm{a}}$ & $360^{\mathrm{a}}$ & 99.44 \\
\hline Control negative & $65000^{\mathrm{b}}$ & $65000^{\mathrm{b}}$ & 0.0 \\
\hline
\end{tabular}

*Tukey $(0.05)=$ Letras iguales $=$ no existe diferencia estadística significativa.

Límite máximo permisible de HC por la NOM-138 SEMARNAT/SS-2003= 4400 ppm

\section{Discusión}

La BR del suelo contaminado con 55000 y 65000 ppm de ARA por BS con la SM/14 semanas fue evidente la disminución de cada concentración; lo que sugiere que los compuestos de nitrógeno $(\mathrm{N})$, fosforo $(\mathrm{P})$ y potasio $(\mathrm{K})$ estimularon la actividad de la microbiota nativa heterotrófica oxidante de la fracción alifática y parcialmente de la aromática del ARA (Vallejo et al. 2005, Gómez et al. 2009). Mientras que en el mismo suelo contaminado con ARA sin BS o control negativo de cada concentración de ARA, la atenuación natural no modificó 
esas concentraciones. En el suelo después la BS con la incorporación de $V$. sativa como $\mathrm{AV}$, se indica que al enriquecerlo con compuestos orgánicos de nitrógeno, fósforo y minerales; aumentaron la actividad de la microbiota nativa para mineralizar el ARA (Asiabadi et al. 2014). En el suelo la degradación del ARA por BS, fue variable al igual que la concentración del $\mathrm{N}$ total, elemento básico que se consumió mas cuando se contamino con 65000 ppm de ARA y menor con 55000 ppm, lo que indica que en el suelo la heterogénea solubilización de las fracciones alifáticas y aromáticas ARA, provocó que su mineralización no fuese proporcional a la concentración original del ARA y del $\mathrm{N}$ total (Graham et al. 1999, Gómez et al. 2009).

En el suelo bioestimulado con la SM y después con la incorporación de $V$. sativa como AV, fue posible enriquecerlo con los minerales básicos suficientes para reequilibrar la relación $\mathrm{C}: \mathrm{N}$, en consecuencia se mejoró la actividad de las raíces de $S$. bicolor para la mayor fitodegradación del ARA, principalmente por la doble inoculación con los MPCV; ya que está demostrado que P. chrysogenum induce la formación de Lacasa un enzima involucrada en el rompimiento de aromáticos similares a los registrados en el ARA (PérezArmendáriz et al. 2011, Asquit et al. 2012). Mientras que se sugiere que a nivel de floración en las raíces de $S$. bicolor se mejoró su actividad metabólica para fitodegradar los $\mathrm{HC}$, en parte porque las raíces generan enzimas involucradas en las síntesis de la lignina, que contribuyen a la mineralización de una parte de la fracción aromática del ARA, lo que a su vez aumentó su resistencia a la fitotoxicidad del ARA. Estos resultados también apoyan que $S$. bicolor fue apoyado por B. cepa- cia y P. chrysogenum microorganismos que transforman exudados de la raíz en sustancias promotoras del crecimiento vegetal, además de que ambos géneros y especies tienen codificado el usar HC como los del ARA como fuentes de carbón y energía, en especial a nivel de su rizosfera, con lo cual potenciaron en S. bicolor una mayor fitodegradación del ARA lo que dio como resultado bajar su concentración a valores menores a la máxima aceptada por la NOM-130-SEMARNAT/SS -2003 para considerar que el mismo suelo contaminados con diferentes y relativamente altas concentraciones de ARA fueron en efecto biorremediados (Dua el al. 2012, Sánchez-Yáñez 2011).

Otro aspecto interesante de la FR del suelo contaminado con ARA es que S. bicolor desarrolla un sistema radical con especial capacidad de penetración al interior del suelo, que mejora algunas de sus propiedades físicas como el drenaje y aireación; al inducir la formación de micro y macro agregados que favorecen los espacios poros de esta matriz heterogénea (Pérez-Armendáriz et al. 2011), lo que facilito una mayor y eficaz mineralización del ARA en su rizosfera por los MPCV que se emplearon para potenciar esta acción depuradora del suelo (Juárez-Cisneros \& Sánchez-Yánez 2014).

La combinación de la biorremediación y fitorremediación con el S. bicolor y los MPCV fue una estrategia integral que permitió la remediación del suelo contaminado con elevados niveles de ARA a valores inferiores al máximo permisible por la NOM-138 SEMARNAT/SS-2003 sin el riesgo de causar un daño ambiental secundario en comparación con los métodos químicos que dejan residuos tóxicos y tienen un valor económico relativamente mayor (USEPA 1991). 


\section{Conflictos de intereses}

Los participantes en esta investigación aseguramos que no existe ningún problema de intereses relacionados con la planeación, ejecución y reporte de esta investigación que comprometa el valor de los resultados obtenidos o sus consecuencias en términos científicos, técnicos o de cualesquier otra índole.

\section{Agradecimientos}

Al proyecto 2.7 (2015) apoyado por la Coordinación Investigación Científica-UMSNH CECTI1004 y BIONUTRA S.A de CV, Maravatio, Mich, México.

\section{Literatura citada}

Asiabadi FI, Mirbagheri SA, Najafi P, Moatar F. Phytoremediaton of petroleum-contami nated soils aroud isahan oil refinery (Iran) by sorghum and barley. Curr World Environ. 2014; 9: 65-72.

Asquith E, Geary M, Nolan A, Evans C. Comparative Bioremediation of Petroleum Hydrocarbon-Contaminated Soil by Biostimulation, Bioaugmentation and Surfactant Addition. J Environ Sci Eng. A.1. 2012; 1: 637-650.

Aurand LW, Woods AE, Wells MR. Food Composition and Analysis. Test of food, USA. 1987; pp. 57-84. [Consultado 12 feb. 2015]. Disponible en http://depa.fquim.unam.

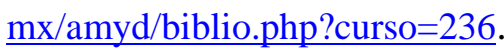

Dua M, Sing A, Sethunathan R, Johri A. Biotechnology and bioremediation: successes and limitations. Appl Microbiol Biotech. 2002; 59: 143-152.

Ferrera-Cerrato R, Alarcón A, Trejo-Aguilar D, Sangabriel W, Mendoza-López M, Cruz-Sánchez J, et al. Fitorremediación de un suelo contaminado con combustóleo utilizando Phaseolus coccineus y fertilización orgánica e inorgánica. Agrociencia. 2007; 41: 817-826.

Gómez W, Gaviria J, Cardona S. Evaluación de la bioestimulación frente a la atenuación natural y la bioaumentación en un suelo contaminado con una mezcla de gasolinadiesel. Dyna. 2009; 76: 83-93.

Graham D W., Smith V H., Law K P., Clealand D., 1999. Effects of nitrogen and phosphorus supply on hexadecane biodegradation in soil systems. Water Air Soil Pollution 111: 1 - 18.

Hernández-Valencia L, Mager D. Uso de $P a$ nicum maximun y Brachiaria brizantha para fitorremediar suelos contaminados con un crudo de petróleo liviano. Bioagro. 2003; 15: 149-155.

Juárez-Cisneros G, Sánchez-Yáñez JM. Biorrestauración de suelo contaminado con aceite residual automotriz por bioestimulación con lombricomposta y fitorremediación con Sorghum vulgare inoculado con Bacillus cereus y Rhizobium etli. J Selva Andina Biosph. 2014; 2:15-22. 
Norma mexicana NMX-F-089-S-1978. Determinación de extracto etéreo (método soxhlet). Gobierno de México. Disponible en línea: http://www.laselva.edu.mx/rcalde ron/wpcontent/uploads/2012/11/Pr\%C3\%A 1ctica-6-1PAL-An\%C3\%A1lisis.pdf. Fecha de último acceso: Noviembre 2014.

Norma oficial mexicana NOM-138-SEMARNAT/ SS-2003, límites máximos permisibles de hidrocarburos en suelos y las especificaciones para su caracterización y remediación. Gobierno de México. Fecha de último acceso: Diciembre 2014.

Pérez-Armendáriz B, Castañeda-Antonio D, Castellanos G, Jiménez-Salgado T, TapiaHernández A, Martínez-Carrera D. Efecto del antraceno en la estimulación del crecimiento en maíz y frijol. Terra Latinoam. 2011; 29: 95-102.

Sánchez-Yáñez JM. Breve Tratado de Microbiología Agrícola, teoría y práctica, Ed. Universidad Michoacana de San Nicolás de Hidalgo, Centro de Investigaciones y Desarrollo del Estado de Michoacán. SEDAGRO, CONSUSTENTA SA de CV, Morelia, Michoacán, México. 2007; 130-133, 136-138, 153-155. pp. ISBN: 978-97095424-1-7.
Sánchez-Yáñez J M. Biorremediación de ambientes impactados con aceites y lubricantes en: Biorremediación, Estrategias contra la contaminación ambiental. 2011. ISBN: 978-1-59754-728-4 pp: 62-75, 79-91. www.librosenred.com.

Smith VH, Graham DW, Clealand DD. Application of resource-ratio theory to hydrocarbon biodegradation. Envirom Sci Technol. 1998; 32: 3386-3395.

USEPA. Understanding bioremediation a guidebook for citizens. EPA/540/2-91/002. Office of Research Development. Washin ton, DC. EUA. 1991.

Vallejo V, Salgado L, Roldan F. Evaluación de la bioestimulación en la biodegradación de TPH en suelos contaminados con petróleo. Rev Colomb Biotecnol. 2005; 7: 6778. 